\title{
Plasma MicroRNA-126-5p is Associated with the Complexity and Severity of Coronary Artery Disease in Patients with Stable Angina Pectoris
}

\author{
Hai-yu Lia Xin Zhaob Yu-Zhou Liua Zhe Menga ${ }^{a}$ Dan Wang ${ }^{a} \quad$ Fan Yang ${ }^{a}$ \\ Qiang-wei Shia \\ aDepartment of Cardiology, The first Affiliated Hospital, Zhengzhou University, Zhengzhou, \\ bDepartment of Cardiology, Beijing Anzhen Hospital, Capital Medical University, Beijing, China
}

\section{Key Words}

Atherosclerosis • Biomarkers • Circulating microRNAs • Coronary heart disease

\begin{abstract}
Background: Coronary artery disease (CAD) is a major problem worldwide. As an endotheliumenriched microRNA (miRNA), miR-126 has been reported to serve as a potential biomarker of acute myocardial infarction. However, the relationship between miR-126 and the severity of CAD remains unknown. This study was designed to test whether circulating miR-126 levels are associated with the severity of CAD. Methods: The present study enrolled 40 patients who had risk factors for CAD without angiographically significant $C A D$, and 110 patients presenting with stable angina pectoris, who were validated left main coronary artery disease (LMCA) and/ or multi-vessel disease by coronary angiography. The expression levels of plasma miR-126-5p from all enrolled subjects were estimated by quantitative real-time polymerase chain reaction (qRT-PCR). Then, the relationships between plasma miR-126-5p levels, number of diseased vessels and the corresponding Synergy between PCI with Taxus and Cardiac surgery (SYNTAX) score were analyzed. Results: The expression of circulating miR-126-5p was affected by some $C A D$ risk factors including aging, dyslipidemia and DM. Furthermore, plasma miR-126-5p levels were significantly down-regulated in CAD patients with multi-vessel disease, higher SYNTAX score, rather than isolated LMCA and low SYNTAX score. Conclusion: Circulating miR-126-5p has emerged as a potential biomarker for complexity and severity of CAD in patients with stable angina pectoris.
\end{abstract}




\section{Introduction}

Coronary artery disease (CAD) represents a major cause of morbidity and mortality worldwide. In recent years, the Synergy between PCI with Taxus and Cardiac surgery (SYNTAX) score was created to predict early and late clinical outcomes, which facilitates to determine the optimal revascularization modality for patients with severe CAD (left main coronary artery disease (LMCA) and/or multi-vessel disease) [1-4]. However, the calculation SYNTAX score is based on angiography results, which is often perceived as difficult and time consuming. Various circulating biomarkers associated with CAD have been identified [5-7], but only few of them provide diagnostic value regarding the severity and complexity of CAD. Therefore, more studies are needed to identify novel biomarkers that assess the severity of CAD.

MicroRNAs (miRNAs) are a class of short (18-22 nt), single-stranded noncoding RNAs that regulate cellular functions through the degradation and translational repression of target mRNAs [8]. miRNAs have been confirmed to be critically involved in various essential biological processes, including proliferation, development, differentiation, and apoptosis [9]. Additionally, circulating miRNAs could be useful as diagnostic or/and prognostic biomarkers for various cardiovascular diseases, such as atrial fibrillation, myocardial infarction (MI), heart failure and cardiac hypertrophy [5, 6, 10-16]. Interestingly, miRNAs have been found in plasma and other body fluids as stable molecules protected from endogenous RNase activity, which suggests that circulating miRNAs might be ideal biomarkers in cardiovascular diseases $[17,18]$.

Atherosclerosis and aberrant thrombosis are the primary pathological changes involved in CAD [19]. Endothelial dysfunction has been confirmed to be critically involved in the development of atherosclerosis [19]. As an endothelial-enriched miRNA, miR-126 has been reported to play important roles in modulating vascular development and angiogenesis [20]. Additionally, circulating miR-126 in the plasma has been reported to be down-regulated in patients with acute myocardial infarction (AMI) [21]. However, there was still a divergence on the expression of circulating miR-126 in patients with CAD in previous studies, which might be due to the involved patients with different severity and complexity of CAD [22, 23]. Furthermore, the expression level of circulating miR-126-5p in patients with severe CAD remains unknown. So herein, we investigated the expression of miR-126-5p in patients with LMCA and/or multi-vessel disease, and determined the relationship between circulating miR-126-5p levels and the corresponding SYNTAX score of severity and complexity of CAD.

\section{Materials and Methods}

\section{Ethics}

This study was approved by the Research Ethics Committee of Zhengzhou University. All participants from the First Affiliated Hospital of Zhengzhou University were enrolled between June 2013 and May 2015. All patients involved in this study received oral and written information regarding the objectives of the study and provided written informed consent.

\section{Population study}

In total, 110 patients who were scheduled for coronary angiography due to stable angina pectoris (SAP), and subsequently who were diagnosed LMCA or/and muti-vessel disease were enrolled in our study. Another 40 patients matched for sex, age, smoking status, hypertension, and diabetes mellitus (DM) without angiographically significant CAD served as controls. Coronary angiograms were obtained at the catheterlaboratory in the Department of Cardiology at the First Affiliated Hospital of Zhengzhou University. The complexity and severity of the CAD was scored when the following criteria were met: stenosis of $\geq 50 \%$ in isolated LMCA or/and muti-epicardial vessels (vessel diameter $>1.5 \mathrm{~mm}$ ). Qualitative Comparative Analysis software was used (QCA, CAAS, Siemens) to measure the percentage of stenosis and the dimension of the vessel. Additionally, a detailed review of each patient's characteristics and medical history was collected to 


\section{Cellular Physiology Cell Physiol Biochem 2016;39:837-846

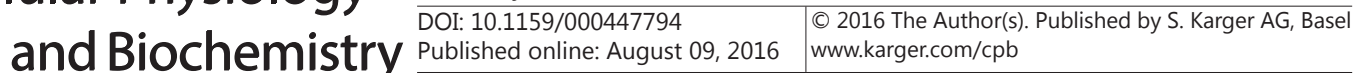 \\ Lie et al.: microRNA-126-5p is Associated with Stable Angina Pectoris}

gather data regarding medications at time of admission and risk factors for CAD, including age, male gender, smoking status, hypertension, DM and dyslipidemia. To minimize the potential influence of other diseases, the general exclusion criteria included the following: a known history of other cardiovascular diseases (e.g., atrial fibrillation, congestive heart failure, unstable angina pectoris (UAP) and MI), a history of either percutaneous coronary intervention (PCI) or coronary artery bypass graft (CABG), severe hepatic or renal dysfunction, recent infection or active chronic inflammatory disease during the last 6 weeks.

Plasma collection and storage

In the present study, peripheral venous blood samples for all participants involved were collected in EDTA-coated tubes when the patients were admitted to the cardiovascular medicine department. All blood samples were then treated with the following protocol: centrifugation at $1500 \times \mathrm{g}$ for $10 \mathrm{~min}$ at $4^{\circ} \mathrm{C}$ to remove large cells and debris. The supernatant was then isolated and centrifuged at $14,000 \times \mathrm{g}$ for 15 $\min$ at $4{ }^{\circ} \mathrm{C}$ to obtain platelet-poor plasma. All plasma samples were stored in aliquots at $-80^{\circ} \mathrm{C}$ until RNA extraction.

\section{MicroRNA analysis}

Total RNA was extracted from plasma using the TRIzol LS Reagent (Invitrogen, US) in accordance with the manufacturer's instructions. After purification from plasma (500 $\mu \mathrm{L})$, total RNA was eluted into $10 \mu \mathrm{L}$ of RNase-free water. Subsequently, quantitative real-time polymerase chain reaction (qRT-PCR) was performed using the Bulge-Loop ${ }^{\mathrm{TM}}$ miRNA qRT-PCR Detection Kit (Ribobio Co., Guangzhou, China) and TransStart $^{\mathrm{TM}}$ Green qPCR SuperMix (Ribobio Co., Guangzhou, China) with Caenorhabditis elegans miR-39 (cel-miR-39) used as the normalization control. Then, qRT-PCR was performed with the ABI 7500 Fast RealTime PCR System according to the manufacturer's protocol. The reactions were incubated at $95^{\circ} \mathrm{C}$ for $30 \mathrm{~s}$, followed by 40 cycles of $95^{\circ} \mathrm{C}$ for $30 \mathrm{~s}, 60^{\circ} \mathrm{C}$ for $20 \mathrm{~s}$, and $70^{\circ} \mathrm{C}$ for $1 \mathrm{~s}$. Finally, the cycle threshold (Ct) values were normalized to cel-miR-39 using the formula $2^{\text {-(Ct [miRNA]-Ct [cel-miRNA-39]) }}$, and the $2^{-\Delta \Delta C t}$ method was used to calculate the relative expression level of miR-126-5p in all samples.

\section{SYNTAX score calculation}

The SYNTAX scoring system was applied to calculate the severity and complexity of the CAD using the online SYNTAX calculator version 2.11 (www.syntaxscore.com). The SYNTAX score was determined by two experienced interventional cardiologists who were blinded to the patient's clinical and laboratory data. Based on previous reports, all enrolled patients were divided into tertiles according to the corresponding SYNTAX score of CAD as follows: low, 0-22; intermediate, 23-32; high, >33.

\section{Statistical analyses}

All data are presented as the means \pm standard deviations (SD) or standard errors (SEM). Continuous variables were compared using either the Mann-Whitney U test or the Kruskal-Wallis test, whereas the one-way ANOVA and Tukey's test were used to compare more than two groups. For categorical variables, either the Chi-Square test or Fisher's test was used appropriately. Univariate linear regression analysis were used to investigate the correlations between the plasma levels of miR-126-5p and CAD traditional risk factors. Additionally, univariate and multivariable linear regression analysis were performed to determine the correlation between plasma miR-126-5p and severity of CAD. For all statistical analyses, SPSS 13.0 was used, and a two-tailed $\mathrm{p}<0.05$ was considered to be significant.

\section{Results}

\section{Participant characteristics}

The basic characteristics of the patients and control subjects are shown in (Table 1). There were no significant differences among CAD patients with different SYNTAX score and the control group for any of the considered variables (i.e., age, male gender, BMI, smoking status, history of DM, total cholesterol, total triglycerides, LDL cholesterol, HDL cholesterol, heart rate, systolic blood pressure, diastolic blood pressure, creatinine and high sensitivity C-reactive protein). However, the prevalence of dyslipidemia history, SYNTAX score and 
Lie et al.: microRNA-126-5p is Associated with Stable Angina Pectoris

Table 1. Baseline characteristics of patients with low, medium and high Syntax score and controls. BMI, body mass index; SBP, systolic blood pressure; DBP, diastolic blood pressure; TC, total cholesterol; TG, total triglyceride; HDL, high-density lipoprotein; LDL, low-density lipoprotein; DM, diabetes mellitus; Hs-CRP, high-sensitivity C-reactive protein; ACEI/ARB angiotensin converting enzyme inhibitor/angiotensin receptor blocker. Data are shown as the mean \pm SD; ${ }^{*} p<0.05$ and ${ }^{* *} p<0.01$ for the CAD groups $v s$. control group

\begin{tabular}{|c|c|c|c|c|c|}
\hline \multirow[t]{2}{*}{ Variables } & Controls & & CAD & & \multirow[t]{2}{*}{$P$ value } \\
\hline & \multicolumn{4}{|c|}{ Syntax Scores } & \\
\hline Participates number & $(n=40)$ & $\leq 22(n=40)$ & $>22$ and $\leq 32(n=40)$ & $>32(n=30)$ & \\
\hline Age (years) & $64.0 \pm 10.4$ & $66.5 \pm 11.7$ & $67.4 \pm 9.7$ & $68.9 \pm 11.3$ & 0.34 \\
\hline Male Gender, n (\%) & $28(70 \%)$ & $26(65 \%)$ & $24(60 \%)$ & $17(57 \%)$ & 0.66 \\
\hline BMI, $\mathrm{kg} / \mathrm{m}^{2}$ & $23.9 \pm 3.5$ & $24.9 \pm 2.7$ & $24.4 \pm 3.0$ & $25.2 \pm 3.2$ & 0.31 \\
\hline Smoker, n (\%) & $10(25 \%)$ & $12(30 \%)$ & $12(30 \%)$ & $13(43 \%)$ & 0.42 \\
\hline Heart rate & $68.4 \pm 7.8$ & $67.5 \pm 9.5$ & $70.4 \pm 8.5$ & $72.4 \pm 11.8$ & 0.14 \\
\hline Hypertension history, n (\%) & $12(30 \%)$ & $16(40 \%)$ & $15(38 \%)$ & $14(47 \%)$ & 0.55 \\
\hline SBP (mmHg) & $128.5 \pm 11.5$ & $132.4 \pm 13.6$ & $131.2 \pm 12.9$ & $129.7 \pm 13.4$ & 0.56 \\
\hline DBP (mmHg) & $78.2 \pm 8.7$ & $80.5 \pm 9.4$ & $79.8 \pm 10.5$ & $82.0 \pm 9.8$ & 0.42 \\
\hline DM, n (\%) & $5(13 \%)$ & $9(23 \%)$ & $11(28 \%)$ & $10(33 \%)$ & 0.19 \\
\hline Dyslipidemia history, n (\%) & $14(47 \%)$ & $22(55 \%)$ & $26(65 \%)$ & $20(67 \%)$ & $<0.01$ \\
\hline $\mathrm{TC}(\mathrm{mmol} / \mathrm{L})$ & $4.2 \pm 0.2$ & $4.4 \pm 0.5$ & $4.3 \pm 0.6$ & $4.5 \pm 0.4$ & 0.21 \\
\hline TG (mmol/L) & $1.7 \pm 0.9$ & $1.9 \pm 0.3$ & $1.9 \pm 0.2$ & $2.0 \pm 0.2$ & 0.09 \\
\hline HDL-C (mmol/L) & $1.0 \pm 0.04$ & $1.0 \pm 0.04$ & $1.0 \pm 0.03$ & $0.98 \pm 0.03$ & 0.06 \\
\hline LDL-C (mmol/L) & $2.7 \pm 0.8$ & $2.6 \pm 0.7$ & $2.6 \pm 0.4$ & $2.5 \pm 0.6$ & 0.65 \\
\hline Creatinine (mg/dl) & $68.4 \pm 12.3$ & $72.4 \pm 16.5$ & $70.7 \pm 14.8$ & $75.5 \pm 15.6$ & 0.25 \\
\hline Hs-CRP (mg/L) & $0.9 \pm 1.5$ & $1.5 \pm 1.9$ & $1.6 \pm 2.1$ & $1.8 \pm 3.5$ & 0.36 \\
\hline Syntax score & / & $12.4 \pm 3.1$ & $26.0 \pm 2.6$ & $35.2 \pm 2.2$ & $<0.01$ \\
\hline \multicolumn{6}{|l|}{ Medications on admission } \\
\hline Aspirin, No. (\%) & $8(20 \%)$ & $30(75 \%)$ & $32(80 \%)$ & $24(80 \%)$ & $<0.01$ \\
\hline Clopidogrel, No. (\%) & / & $7(18 \%)$ & $5(13 \%)$ & $5(17 \%)$ & $<0.01$ \\
\hline Beta-blockers, No. (\%) & $6(15 \%)$ & $17(43 \%)$ & $22(55 \%)$ & $16(53 \%)$ & $<0.01$ \\
\hline ACEI/ARB, No. (\%) & $7(18 \%)$ & $20(50 \%)$ & $25(63 \%)$ & $18(60 \%)$ & $<0.01$ \\
\hline Statins, No. (\%) & $12(30 \%)$ & $21(53 \%)$ & $26(65 \%)$ & $20(67 \%)$ & $<0.01$ \\
\hline
\end{tabular}

medication on admission (including aspirin, clopidogrel, beta-blockers, ACEI/ARB and statins) were significantly different between these groups.

Association of plasma miR-126-5p
levels and traditional risk factors of
atherosclerosis
It is well established that many traditional risk factors (e.g., gender, age, smoking, DM, BMI or hypertension) play important roles in the development of atherosclerosis. To further investigate whether these traditional risk factors impact the expression of circulating miR-126-5p in patients with severe CAD, we performed a univariate linear regression analysis.

The results showed that some risk factors including aging, DM and hyperlipidemia were negatively associated with the down-regulation of circulating miR-126-5p $(p=0.043,0.008$
Table 2. Univariate linear regression analysis between traditional cardiovascular diseases and the plasma levels of miRNA-126-5p. BMI, body mass index; DM, diabetes mellitus; Data are shown as the mean \pm SEM; ${ }^{*} p<0.05$ and ${ }^{* *} p<0.01$

\begin{tabular}{llll}
\hline Variables & Estimate & SEM & P value \\
\hline Age & -0.004 & 0.002 & $0.043^{*}$ \\
Male gender & 0.087 & 0.078 & 0.267 \\
BMI & 0.004 & 0.007 & 0.552 \\
DM & -0.127 & 0.072 & $0.008^{* *}$ \\
Hypertension & -0.036 & 0.039 & 0.351 \\
Dyslipidemia & -0.196 & 0.076 & $0.011^{*}$ \\
Smoker & -0.006 & 0.047 & 0.891 \\
\hline
\end{tabular}




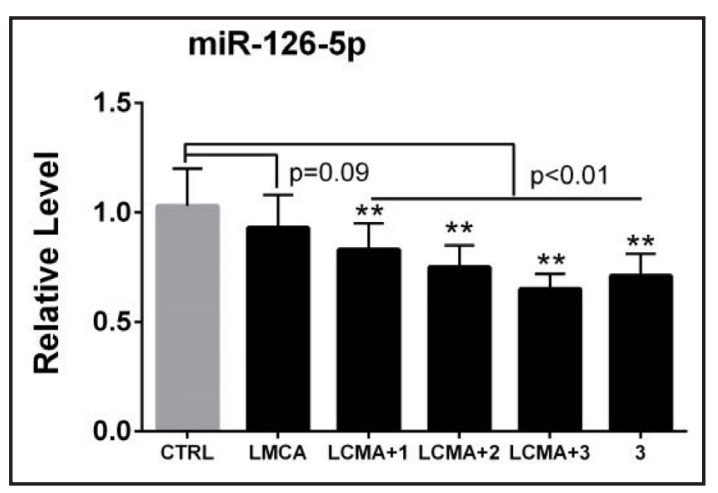

Fig. 1. Association between plasma miRNA-126-5p level and the diseased vessel in severe CAD patients presenting with stable angina pectoris and control subjects. The expression level of plasma miR-126 in control groups and patients with isolated LMCA; LMCA plus single-, double- or triple-vessel disease and triple-vessel disease alone. CAD, coronary artery disease; LMCA, left main coronary artery; CTRL, control group. The data are shown as the mean \pm SD; ${ }^{* *} p<0.01$. ${ }^{*} p<0.05$ and ${ }^{* *} p<0.01$ for the CAD groups $v s$. the control group.

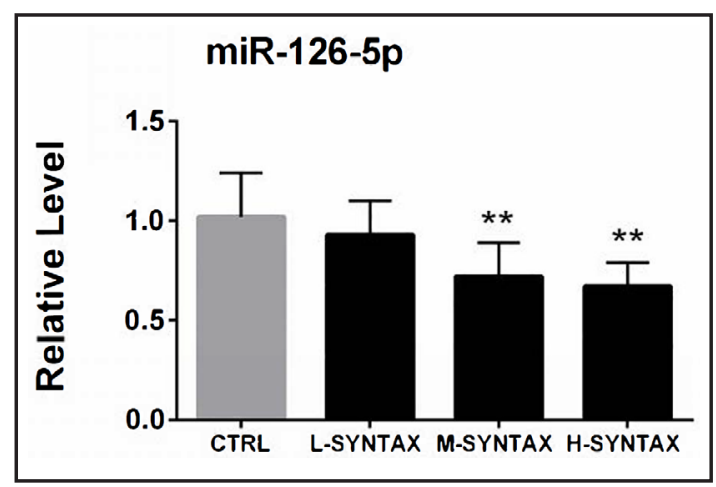

Fig. 2. Association between plasma miRNA-126$5 p$ level and the SYNTAX score in SAP patients with coronary artery disease. SYNTAX score, Synergy between PCI with Taxus and Cardiac surgery score; L-SYNTAX, low SYNTAX score group; M-SYNTAX, intermediate SYNTAX score group; H-SYNTAX, high SYNTAX score group. The data are shown as the mean $\pm \mathrm{SD} ;{ }^{*} p<0.05$ and ${ }^{* *} p<0.01$ for the CAD groups $v s$. the control group.

Table 3. Association of miRNA-126-5p with the severity and complexity of coronary artery disease. SYNTAX score, Synergy between PCI with Taxus and Cardiac surgery score Data are shown as the mean \pm SEM; ${ }^{* *} p<0.01$

\begin{tabular}{lllllll}
\hline & \multicolumn{3}{c}{ Univariate } & \multicolumn{2}{c}{ Adjusted for traditional risk factors } \\
\hline & Estimate & SEM & P value & Estimate & SEM & P value \\
SYNTAX score & -0.011 & 0.002 & $<0.001^{* *}$ & -0.011 & 0.002 & $<0.001^{* *}$ \\
\hline
\end{tabular}

and 0.011, respectively) (Table 2). However, male gender, BMI, hypertension and smoking were not significantly associated with the down-regulation of plasma miR-126-5p levels ( $\mathrm{p}=0.267,0.552,0.351$ and 0.891 , respectively) (Table 2).

Down-regulation of plasma miR-126-5p levels is associated with the severity of CAD

Based on the diseased vessel according to their coronary angiography results, the 110 CAD patients were categorized as follows: 26 patients with an isolated LMCA (24\%), 22 patients with LMCA plus single-vessel disease (20\%), 20 patients with LMCA plus doublevessel disease (18\%), 15 patients with LMCA plus triple-vessel disease (14\%) and 27 patients with three-vessel disease (25\%). The expression level of miR-126-5p in patients with multi-vessel disease was lower than the control group (Fig. 1). However, the expression of circulating miR-126-5p was not dramatically down-regulated in patients with an isolated LMCA compared with the control subjects.

\section{Association of plasma miR-126-5p levels with the SYNTAX score}

To further investigate the association between the expression of plasma miR-126-5p and the severity and complexity of CAD, we determined the association of circulating miR126-5p with the corresponding SYNTAX score of the diseased vessels. Interestingly, there was no significant difference in the expression of circulating miR-126-5p between CAD patients with low SYNTAX score and control subjects. However, compared to controls, the expression of miR-126-5p was significantly lower in CAD patients with intermediate or high SYNTAX score (Fig. 2). Moreover, the results both of the univariate and multivariate linear regression 
analyses after adjustment for traditional coronary risk factors showed that a higher SYNTAX score was significantly associated with lower miR-126-5p levels (estimate, -0.011; standard error, 0.002; $\mathrm{p}<0.001$ and estimate, -0.011; standard error, 0.002; $\mathrm{p}<0.001$, respectively) (Table 3). Meanwhile, plasma miR-126-5p levels were significantly negatively correlated with the SYNTAX score $(\mathrm{r}=0.511, \mathrm{p}=-0.001)$, suggesting that the reduced expression of plasma miR-126-5p is significantly associated with more severe and complex CAD (Fig. 3).

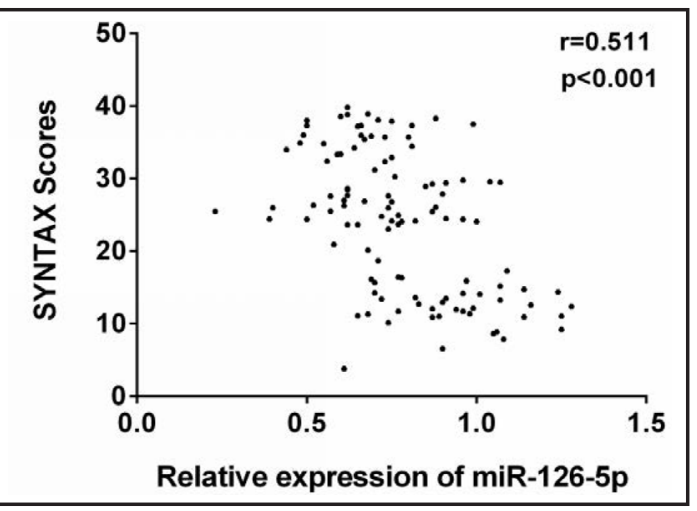

Fig. 3. The correlation between plasma miRNA-126 and the SYNTAX score. SYNTAX score, Synergy between PCI with Taxus and Cardiac surgery score.

\section{Discussion}

In the present study, we found that plasma miR-126-5p was significantly down-regulated in patients with severe CAD. Furthermore, some traditional cardiovascular disease risk factors, such as aging, DM and dyslipidemia should be responsible for the down-regulation of plasma miR-126-5p in CAD patients. Compared with control subjects, the expression of plasma miR-126-5p was significantly lower in CAD patients with either intermediate or high SYNTAX score, instead of low SYNTAX score. Additionally, plasma miR-126-5p was significant negative correlation with the SYNTAX score of diseased vessel. According to the above findings, our present study indicated that circulating miR-126-5p might be as a novel predictive biomarker for the severity and complexity of coronary artery lesions in patients with SAP.

Atherosclerosis is currently defined as a chronic inflammatory disease of the vascular wall, which constitutes basic pathology changes of cardiovascular and cerebrovascular diseases, such as MI, ischemic stroke, peripheral arterial disease and carotid plaques [19]. Some substantial changes contribute to the pathological changes of atherosclerosis, including dysfunction of vascular wall cells [i.e., endothelial cells (ECs) and vascular smooth muscle cells (VSMCs)] and the recruitment of leukocytes [24]. According to previous studies, dysfunctional ECs play a pivotal role in atherosclerosis by impairing endotheliumdependent vasodilatation, increasing vascular permeability, up-regulating the expression of chemokines/adhesion molecules and decreasing endothelial regeneration [24]. So, some biomarkers which impact on endothelial function might reflect the condition of atherosclerosis.

In recent years, emerging evidence has indicated that miRNAs play important roles in regulating endothelial function and atherosclerosis [25]. As one of the most abundant miRNAs in ECs, miR-126-5p plays a crucial anti-atherogenic role by regulating the function of ECs and enhancing endothelial repair [26]. miR-126-5p deficiency led to leaky vessels and hemorrhage due to the loss of vascular integrity during embryonic development, which is partly mediated by direct targeting of negative regulators of vascular endothelial growth factor (VEGF) signaling, such as sprout-related proteins and phosphoinositol-3 kinase regulatory subunit 2 [27]. In addition, Harris et al. [28] reported that miR-126-5p negatively regulates leukocyte adherence to ECs by targeting endothelial vascular cell adhesion molecule-1 (VCAM-1) in ECs. A study by Van Solingen et al. showed that transferring ECderived apoptotic micro-vesicles containing miR-126-5p into atherosclerotic lesions significantly ameliorates the progression of atherosclerosis through the recruitment of Sca1-positive EC progenitor cells [29]. Meanwhile, miR-126-5p also could promote endothelial proliferation and limit atherosclerosis by suppressing Notch1 inhibitor delta-like 1 homolog (Dlk1) [30]. 
It has been established that miR-126-5p is significantly down-regulated in human atherosclerotic lesions [30]. Meanwhile, circulating miR-126-5p also is dramatically downregulated in patients with acute coronary syndrome (ACS), including AMI and UAP [21, 31]. In a recent study, Fichtlscherer et al. reported that plasma miR-126 was down-regulation in patients with CAD [22]. However, Sun et al. found that plasma was not down-regulation in patients with CAD [23]. We assumed that the different plaque burden of diseased vessel in the enrolled participants might be responsible for the discrepancy findings among these studies. Thus, for the first time, we investigated the predictive value of plasma circulating miR-126$5 p$ for the severity and complexity of CAD. Our data indicated that not only miR-126-5p is critically involved in the regulation of $\mathrm{EC}$ function and plaque formation, but circulating miR126-5p levels can predict the severity of atherosclerosis lesions in CAD patients presenting with SAP.

Our data demonstrated that the expression of circulating miR-126-5p could also be influenced by some traditional cardiovascular risk factors, including aging, DM and hyperlipidemia, which is in accordance with previous studies [23, 32-34]. It is worth noting that although these risk factors have been reported to be critically involved in the formation of atherosclerosis, circulating miR-126-5p is a potential independent predictive factor for the severity of cardiovascular artery disease.

In recent years, the SYNTAX score was created to evaluate the severity and complexity of CAD, which facilitates the identification of the optimal strategy for patients with severe CAD $[1,3]$. Currently, the optimal strategy for patients with LMCA and/or multiple vessel disease is mainly based on angiography results, which has many clinical advantages [35]. However, one major limitation is that the calculation of the SYNTAX score is often perceived as difficult and time consuming. We are committed to identifying biomarkers that can accurately reflect the severity and complexity of coronary vessel lesion in patients with CAD. An increasing number of biomarkers (e.g., hemoglobin A1, decoy receptor 3 and serum endocan) may serve as independent predictors for CAD and its severity [36-38]. Regarding miRNAs, a recent study by Gao et al. [39] reported that circulating miR-145, as a VSMC-enriched miRNA that plays an important role in atherosclerosis, was negatively associated with the SYNTAX score and the severity of coronary artery lesion in CAD patients. Our present study indicates that miR-126-5p could serve as another potential miRNA biomarker for the severity and complexity of CAD, which might facilitate the identification of optimal treatment strategies for patients with highly complex coronary artery disease. However, we noticed that the expression of miR-126-5p was neither significantly down-regulated in SAP patients with isolated LMCA nor in patients with low SYNTAX score compared to the controls, which might be due to that the down-regulation of circulating miR-126-5p must reach a threshold plaque burden of diseased vessels.

Interestingly, a recent study reported that up-regulation of miR-126 improved cardiac vascular density and function of monocrotaline-induced pulmonary arterial hypertension $(\mathrm{PAH})$ rats [40]. However, in future, more studies should be used to investigate whether upregulation of circulating miR-126 (in vivo) improve endothelial function, stabilize vulnerable plaque, which might provide a potential therapeutic for the severity and complextity of CAD.

\section{Limitations}

There may be several limitations in the present study. First, as a single center study with a small sample size, the predictive value should be interpreted with caution. Larger clinical studies are definitely required to determine the clinical diagnosis value of miR-126-5p on the severity and complexity of CAD. Second, more studies should be performed to investigate the underlining mechanisms of the association between down-regulated circulating miR-126-5p and severity of CAD. Third, our present data focus on the predictive value of circulating miR126-5p for the severity of CAD in patients with SAP. However, more studies are necessary to investigate the divergence expression of miR-126-5p in patients with AMI/UAP and the

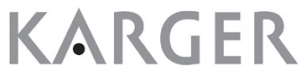




\section{Cellular Physiology Cell Physiol Biochem 2016;39:837-846 \begin{tabular}{ll|l} 
DOI: 10.1159/000447794 & O 2016 The Author(s). Published by S. Karger AG, Basel \\
www.karger.com/cpb
\end{tabular} \\ Lie et al.: microRNA-126-5p is Associated with Stable Angina Pectoris}

severity of CAD in patients with SAP. Finally, there is no doubt that the cardiac/skeletaldrived miRNAs including miR-208, miR-499, miR-1 and miR-133 are critically involved in the CAD, and which have been reported as potential biomarkers for AMI. However, the main pathological changes are endothelial dysfunction and atherosclerotic plaque rather than myocardial necrosis and cell apoptosis in CAD patients presenting with SAP. So, herein, our present study focus on the endothelial-enriched miRNA rather than the widely reported cardiac-specific miRNAs (eg. miR-208, -499, -1 and -133), which clinical value should be investigated by more studies.

\section{Conclusion}

Overall, our present study provides the first evidence that plasma miR-126-5p levels are negatively associated with SAP patients who have severe and complex coronary artery disease. This knowledge may ultimately facilitate the selection of an optimal treatment strategy for the patients.

\section{Disclosure Statement}

The authors have no disclosures to report.

\section{References}

1 Capodanno D, Capranzano P, Di Salvo ME, Caggegi A, Tomasello D, Cincotta G, Miano M, Patane M, Tamburino C, Tolaro S, Patane L, Calafiore AM, Tamburino C: Usefulness of SYNTAX score to select patients with left main coronary artery disease to be treated with coronary artery bypass graft. JACC Cardiovasc Interv 2009;2:731-738.

2 Valgimigli M, Serruys PW, Tsuchida K, Vaina S, Morel MA, van den Brand MJ, Colombo A, Morice MC, Dawkins K, de Bruyne B, Kornowski R, de Servi S, Guagliumi G, Jukema JW, Mohr FW, Kappetein AP, Wittebols K, Stoll HP, Boersma E, Parrinello G: Cyphering the complexity of coronary artery disease using the syntax score to predict clinical outcome in patients with three-vessel lumen obstruction undergoing percutaneous coronary intervention. AM J Cardiol 2007;99:1072-1081.

3 Sianos G, Morel MA, Kappetein AP, Morice MC, Colombo A, Dawkins K, van den Brand M, Van Dyck N, Russell ME, Mohr FW, Serruys PW: The SYNTAX Score: An angiographic tool grading the complexity of coronary artery disease. Euro Intervention 2005;1:219-227.

4 Osnabrugge RL, Magnuson EA, Serruys PW, Campos CM, Wang K, van Klaveren D, Farooq V, Abdallah MS, Li H, Vilain KA, Steyerberg EW, Morice MC, Dawkins KD, Mohr FW, Kappetein AP, Cohen DJ: Costeffectiveness of percutaneous coronary intervention versus bypass surgery from a Dutch perspective. Heart 2015;101:1980-1988.

5 Widmer RJ, Lerman LO, Lerman A: MicroRNAs: Small molecule, big potential for coronary artery disease. Eur Heart J 2016

6 Berezin A, Zulli A, Kerrigan S, Petrovic D, Kruzliak P: Predictive role of circulating endothelial-derived microparticles in cardiovascular diseases. Clin Biochem 2015;48:562-568.

7 Sato K, Watanabe R, Itoh F, Shichiri M, Watanabe T: Salusins: Potential use as a biomarker for atherosclerotic cardiovascular diseases. Int J Hypertens 2013;2013:965140.

8 van Rooij E: The art of microRNA research. Circ Res 2011;108:219-234.

9 Bartel DP: MicroRNAs: Genomics, biogenesis, mechanism, and function. Cell 2004;116:281-297.

10 Romaine SP, Tomaszewski M, Condorelli G, Samani NJ: MicroRNAs in cardiovascular disease: An introduction for clinicians. Heart 2015;101:921-928.

11 Thum T, Condorelli G: Long noncoding RNAs and microRNAs in cardiovascular pathophysiology. Circ Res 2015;116:751-762. 


\section{Cellular Physiology Cell Physiol Biochem 2016;39:837-846 \begin{tabular}{l|l|l} 
and Biochemistry 10.1159/000447794 & $\begin{array}{l}\text { C) 2016 The Author(s). Published by S. Karger AG, Basel } \\
\text { www.karger.com/cpb }\end{array}$ \\
\hline Published online: August 09, 2016
\end{tabular}}

Lie et al.: microRNA-126-5p is Associated with Stable Angina Pectoris

12 Vegter EL, van der Meer P, de Windt LJ, Pinto YM, Voors AA: MicroRNAs in heart failure: From biomarker to target for therapy. Eur J Heart Fail 2016;18:457-48.

13 Tijsen AJ, Pinto YM, Creemers EE: Circulating microRNAs as diagnostic biomarkers for cardiovascular diseases. Am J Physiol Heart Circ Physiol 2012;303:H1085-H1095.

14 Li S, Fan Q, He S, Tang T, Liao Y, Xie J: MicroRNA-21 negatively regulates Treg cells through a TGF-beta1/ Smad-independent pathway in patients with coronary heart disease. Cell Physiol Biochem 2015;37:866878.

15 Xu Y, Zhu W, Sun Y, Wang Z, Yuan W, Du Z: Functional network analysis reveals versatile MicroRNAs in human heart. Cell Physiol Biochem 2015;36:1628-1643.

16 Zhao X, Wang K, Liao Y, Zeng Q, Li Y, Hu F, Liu Y, Meng K, Qian C, Zhang Q, Guan H, Feng K, Zhou Y, Du Y, Chen Z: MicroRNA-101a inhibits cardiac fibrosis induced by hypoxia via targeting TGFbetaRI on cardiac fibroblasts. Cell Physiol Biochem 2015;35:213-226.

17 Dumache R, Ciocan V, Muresan C, Rogobete AF, Enache A: Circulating MicroRNAs as promising biomarkers in forensic body fluids identification. Clin Lab 2015;61:1129-1135.

18 Kondkar AA, Abu-Amero KK: Utility of circulating microRNAs as clinical biomarkers for cardiovascular diseases. Biomed Res Int 2015;2015:821823.

19 Weber C, Noels H: Atherosclerosis: Current pathogenesis and therapeutic options. Nat Med 2011;17:14101422.

20 Wang S, Aurora AB, Johnson BA, Qi X, McAnally J, Hill JA, Richardson JA, Bassel-Duby R, Olson EN: The endothelial-specific microRNA miR-126 governs vascular integrity and angiogenesis. Dev Cell 2008;15:261-271.

21 Long G, Wang F, Duan Q, Chen F, Yang S, Gong W, Wang Y, Chen C, Wang DW: Human circulating microRNA-1 and microRNA-126 as potential novel indicators for acute myocardial infarction. Int J Biol Sci 2012;8:811818.

22 Fichtlscherer S, De Rosa S, Fox H, Schwietz T, Fischer A, Liebetrau C, Weber M, Hamm CW, Roxe T, MullerArdogan M, Bonauer A, Zeiher AM, Dimmeler S: Circulating microRNAs in patients with coronary artery disease. Circ Res 2010;107:677-684.

23 Sun X, Zhang M, Sanagawa A, Mori C, Ito S, Iwaki S, Satoh H, Fujii S: Circulating microRNA-126 in patients with coronary artery disease: Correlation with LDL cholesterol. Thromb J 2012;10:16.

24 Lu H, Daugherty A: Atherosclerosis. Arterioscler Thromb Vasc Biol 2015;35:485-491.

25 Wei Y, Nazari-Jahantigh M, Neth P, Weber C, Schober A: MicroRNA-126, -145, and -155: A therapeutic triad in atherosclerosis? Arterioscler Thromb Vasc Biol 2013;33:449-454.

26 Mocharla P, Briand S, Giannotti G, Dorries C, Jakob P, Paneni F, Luscher T, Landmesser U: AngiomiR-126 expression and secretion from circulating CD34(+) and CD14(+) PBMCs: Role for proangiogenic effects and alterations in type 2 diabetics. Blood 2013;121:226-236.

27 Fish JE, Santoro MM, Morton SU, Yu S, Yeh RF, Wythe JD, Ivey KN, Bruneau BG, Stainier DY, Srivastava D: MiR-126 regulates angiogenic signaling and vascular integrity. Dev Cell 2008;15:272-284.

28 Harris TA, Yamakuchi M, Ferlito M, Mendell JT, Lowenstein CJ: MicroRNA-126 regulates endothelial expression of vascular cell adhesion molecule 1. Proc Natl Acad Sci USA 2008;105:1516-1521.

29 van Solingen C, de Boer HC, Bijkerk R, Monge M, van Oeveren-Rietdijk AM, Seghers L, de Vries MR, van der Veer EP, Quax PH, Rabelink TJ, van Zonneveld AJ: MicroRNA-126 modulates endothelial SDF-1 expression and mobilization of Sca-1(+)/Lin(-) progenitor cells in ischaemia. Cardiovasc Res 2011;92:449-455.

30 Schober A, Nazari-Jahantigh M, Wei Y, Bidzhekov K, Gremse F, Grommes J, Megens RT, Heyll K, Noels H, Hristov M, Wang S, Kiessling F, Olson EN, Weber C: MicroRNA-126-5p promotes endothelial proliferation and limits atherosclerosis by suppressing Dlk1. Nat Med 2014;20:368-376.

31 Ren J, Zhang J, Xu N, Han G, Geng Q, Song J, Li S, Zhao J, Chen H: Signature of circulating microRNAs as potential biomarkers in vulnerable coronary artery disease. Plos One 2013;8:e80738.

32 Olivieri F, Bonafe M, Spazzafumo L, Gobbi M, Prattichizzo F, Recchioni R, Marcheselli F, La Sala L, Galeazzi R, Rippo MR, Fulgenzi G, Angelini S, Lazzarini R, Bonfigli AR, Bruge F, Tiano L, Genovese S, Ceriello A, Boemi M, Franceschi C, Procopio AD, Testa R: Age- and glycemia-related miR-126-3p levels in plasma and endothelial cells. Aging (Albany NY) 2014;6:771-787.

33 Zhang T, Li L, Shang Q Lv C, Wang C, Su B: Circulating miR-126 is a potential biomarker to predict the onset of type 2 diabetes mellitus in susceptible individuals. Biochem Biophys Res Commun 2015;463:60-63. 


\section{Cellular Physiology Cell Physiol Biochem 2016;39:837-846

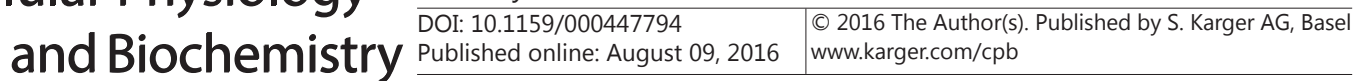 \\ Lie et al.: microRNA-126-5p is Associated with Stable Angina Pectoris}

34 Zampetaki A, Kiechl S, Drozdov I, Willeit P, Mayr U, Prokopi M, Mayr A, Weger S, Oberhollenzer F, Bonora E, Shah A, Willeit J, Mayr M: Plasma microRNA profiling reveals loss of endothelial miR-126 and other microRNAs in type 2 diabetes. Circ Res 2010;107:810-817.

35 Mohr FW, Morice MC, Kappetein AP, Feldman TE, Stahle E, Colombo A, Mack MJ, Holmes DJ, Morel MA, Van Dyck N, Houle VM, Dawkins KD, Serruys PW: Coronary artery bypass graft surgery versus percutaneous coronary intervention in patients with three-vessel disease and left main coronary disease: 5-Year followup of the randomised, clinical SYNTAX trial. Lancet 2013;381:629-638.

36 Chang TY, Hsu CY, Huang PH, Chiang CH, Leu HB, Huang CC, Chen JW, Lin SJ: Usefulness of circulating decoy receptor 3 in predicting coronary artery disease severity and future major adverse cardiovascular events in patients with multivessel coronary artery disease. Am J Cardiol 2015;116:1028-1033.

37 Arbel Y, Zlotnik M, Halkin A, Havakuk O, Berliner S, Herz I, Rabinovich I, Keren G, Bazan S, Finkelstein A, Banai S: Admission glucose, fasting glucose, HbA1c levels and the SYNTAX score in non-diabetic patients undergoing coronary angiography. Clin Res Cardiol 2014;103:223-227.

38 Kose M, Emet S, Akpinar TS, Kocaaga M, Cakmak R, Akarsu M, Yuruyen G, Arman Y, Tukek T: Serum endocan level and the severity of coronary artery disease: A pilot study. Angiology 2015;66:727-731.

39 Gao H, Guddeti RR, Matsuzawa Y, Liu LP, Su LX, Guo D, Nie SP, Du J, Zhang M: Plasma levels of microRNA-145 are associated with severity of coronary artery disease. Plos One 2015;10:e123477.

40 Potus F, Ruffenach G, Dahou A, Thebault C, Breuils-Bonnet S, Tremblay E, Nadeau V, Paradis R, Graydon C, Wong R, Johnson I, Paulin R, Lajoie AC, Perron J, Charbonneau E, Joubert P, Pibarot P, Michelakis ED, Provencher S, Bonnet S: Downregulation of MicroRNA-126 contributes to the failing right ventricle in pulmonary arterial hypertension. Circulation 2015;132:932-943. 